\title{
Cognitive-behavioural therapy for body dysmorphic disorder
}

\author{
David Veale
}

The DSM-IV classification of body dysmorphic disorder (BDD) refers to an individual's preoccupation with an 'imagined' defect in his or her appearance or markedly excessive concern with a slight physical anomaly (American Psychiatric Association, 1994). An Italian psychiatrist, Morselli, first used the term 'dysmorphophobia' in 1886, although it is now falling into disuse, probably because ICD-10 (World Health Organization, 1992) has discarded it, subsuming the condition under hypochondriacal disorder.

The most common preoccupations are with the nose, skin, hair, eyes, eyelids, mouth, lips, jaw and chin. However, any part of the body may be involved and the preoccupation is frequently focused on several body parts. Complaints typically involve perceived or slight flaws on the face, the size of body features (too small or too big), hair thinning, acne, wrinkles, scars, vascular markings, paleness or redness of the complexion, asymmetry or lack of proportion. Sometimes the complaint is extremely vague; it may amount to no more than the patient feeling generally ugly.

\section{Presentation}

Boddy dysmorphic disorder has never been included in the large catchment-area surveys of psychiatric morbidity. However, one Italian study of somatoform disorders found a 1-year prevalence of BDD of nearly 1\% (Faravelli et al, 1997). This would make it relatively common, but mental health professionals do not often diagnose and treat patients with BDD. It is a hidden disorder for which many people do not seek help. There is therefore a low level of awareness of BDD among both the public and health professionals. When people do seek help they are likely to consult a dermatologist or cosmetic surgeon. For example, Sarwer et al (1998) found that $5 \%$ of women presenting at a cosmetic surgery clinic in the USA had BDD. If people with BDD do seek help from a general practitioner or mental health professional, they are often too ashamed to reveal their main symptoms and present with symptoms of depression or social phobia: only specific questioning reveals BDD. Patients are especially secretive about symptoms such as mirrorgazing, probably because they fear they will be thought vain or narcissistic.

On average, BDD is diagnosed 10 years after first presentation and it is often treated inappropriately with antipsychotic medication (Phillips, 1998). Psychotherapists may have little experience in treating BDD patients or lack an effective treatment model. Two randomised controlled trials (RCTs) have been conducted in BDD for cognitivebehavioural therapy (CBT) against a waiting list (Rosen et al, 1995; Veale et al, 1996a) and several case series (Neziroglu \& Yaryura Tobias, 1993; Gomez Perez et al, 1994; Wilhelm et al, 1999). Evidence exists for the efficacy of selective serotonin reuptake inhibitors (SSRIs) in the treatment of BDD (Hollander et al, 1999); this is discussed below.

\section{General assessment}

Beliefs about appearance (for example, that one's nose is too crooked) may be held with poor insight

David Veale is an honorary senior lecturer at the Royal Free Hospital and University College Medical School, University College London and a consultant psychiatrist at The Priory Hospital North London (Grovelands House, The Bourne, Southgate, London N10 3NA. E-mail: David@veale.co.uk). His main interests are in cognitive-behavioural therapy and its application to anxiety disorders. 
(having overvalued ideas) or no insight (being delusional). Within the DSM-IV classification of BDD, the strength of such beliefs is used to determine whether there is an additional (or in ICD-10, an alternative) diagnosis of delusional disorder. The DSM-IV diagnostic criteria state that if preoccupation with a minor physical anomaly is present then the person's concern is regarded as 'markedly excessive'. To distinguish BDD from normal concerns about appearance (especially during adolescence), a preoccupation must also cause significant distress or handicap. Phillips (1996) suggests that to justify a diagnosis of BDD, preoccupation with 'imagined' defects in appearance should last at least 1 hour a day.

Quality of life measures found a degree of distress in BDD that is worse than that in depression (Phillips, 2000). People with the disorder are often unemployed or disadvantaged at work, housebound or socially isolated because of their handicap. A risk assessment must be done, as there is a high rate of suicide and self-harm (Veale et al, 1996b) and 'do-ityourself' cosmetic surgery (Veale, 2000). There is frequent comorbidity, with secondary diagnoses of depression, social phobia, obsessive-compulsive disorder or personality disorder (Veale et al, 1996b). Not surprisingly, BDD patients are difficult to engage and treat.

\section{Cognitive-behavioural assessment}

A cognitive-behavioural assessment uses a threesystems analysis, concentrating on the factors that maintain the disorder, in particular, beliefs and behaviours.

\section{Beliefs}

Patients are often dissatisfied with many areas of their body. Asking them to complete a checklist of different parts of the body, saying exactly what they believe is defective about each part, how they would like it to be and the proportion of distress that is causes can clarify their concerns. The nature of the preoccupation may fluctuate over time, which may explain why, after cosmetic surgery, a preoccupation often shifts to another area of the body.

The next step is to assess the personal meaning or the assumptions held about the perceived defect or ugliness. Patients may have difficulty in articulating the meaning, and a 'downward-arrow technique' can usually identify such assumptions; after eliciting the most dominant emotion associated with thinking about the defect, the therapist asks what is the most shameful (or anxiety-provoking) aspect about the defect. For example, one patient might believe that having a defect affecting his nose will mean that he will end up alone and unloved. Another might believe that the most disgusting aspect of flaws in her skin is that they make her look dirty. These assumptions are then used in cognitive restructuring and behavioural experiments.

The values most important to the individual should also be identified. In BDD, appearance is almost always the dominant and idealised value and the means of defining the self. Patients implicitly view themselves as 'aesthetic objects'. Other important values in BDD include perfectionism, symmetry and social acceptance, and they may take the form of certain rules, for example "I have to be symmetrical".

\section{Behaviours}

Mirror-gazing is at the core of BDD and it appears to be a complex series of safety behaviours. However, mirror-gazing is not even described in standard textbooks of psychopathology. Why do some BDD patients spend many hours in front of a mirror when it invariably makes them feel more distressed and self-conscious? A colleague and I recently conducted a study comparing mirror-gazing in patients with BDD and in normal controls (Veale \& Riley, 2001). We concluded that patients' main motivations for mirror-gazing are: the hope that they will look different; the desire to know exactly how they look; to see how well efforts at camouflage have worked; and a belief that they will feel worse if they resist gazing (although gazing in fact increases distress). Patients were more likely to focus their attention on an internal impression or feeling (rather than on their reflection in the mirror) and on specific parts of their appearance. Although both patients and controls used the mirror for normal actions (to put on make up, shave, groom their hair or check their appearance), only patients performed 'mental cosmetic surgery' to change their body image and practised pulling different faces. A detailed assessment of patients' behaviour in front of a mirror and their motivation is of great value for therapy and for the construction of behavioural experiments to test out beliefs. For example, the duration of the longest mirror-gazing session and the frequency of the shorter sessions can be used throughout therapy to monitor the severity of the behaviour. Other reflective surfaces, such as the back of compact disks and shop window-panes, may also be used, which distort the body image in reality. 
Patients may also check their appearance by measuring their perceived defect, by feeling the contours of the skin or by taking frequent photographs or video recordings of themselves. Other behaviours include asking others to verify the existence of the defect or the effectiveness of camouflage; comparing current appearance with old photographs or with the apperance of other people; wearing make-up 24 hours a day; excessive grooming of the hair; excessive cleansing of the skin; use of facial peelers, saunas or exercises to improve facial muscle tone; beauty treatments (e.g. collagen injections to the lips); cosmetic surgery; and dermatological treatments. There may also be impulsive behaviours such as skin-picking, which produce a very brief sense of satisfaction or pleasure (similar to trichotillomania) followed by a sense of despair and anger.

\section{Social avoidance and anxiety}

Beliefs about being defective and the importance of appearance will drive varying degrees of social anxiety and avoidance. Thus, depending on the nature of their beliefs, patients will tend to avoid a range of public or social situations or intimate relationships. These should be assessed in detail and rated on a scale of 0 to 100 in terms of degree of distress or anxiety endured without any safety behaviours or alcohol. Many patients endure social situations only if they use camouflage or various safety behaviours. These are often idiosyncratic and depend on the perceived defect and cultural norms. Behaviours such as avoidance of eye contact and using long hair or excessive make-up for camouflage are obvious, but others are more subtle and are difficult to detect without quizzing the patient on behaviour in particular social situations. For example, a patient preoccupied with his nose avoided showing his profile in social situations and only stood face on to another. A patient preoccupied with perceived blemishes under her eye wore spectacles to hide the skin. Safety behaviours contribute to the inability to disprove beliefs and further checking in mirrors to see whether the camouflage is 'working'. They must therefore be addressed so that patients can learn to enter public and social situations without them.

Most people with BDD show a slightly greater concern with their own evaluation of themselves than how others evaluate them. They therefore value both aesthetics and social acceptance. A few people with the disorder, however, are almost entirely concerned with the fear of negative evaluation by others (and not with an internal aesthetic standard). These people tend to resemble individuals with social phobia and may be easier to treat than those preoccupied with their own internal aesthetic standards. And a few are almost entirely concerned with meeting a self-judged aesthetic standard and have much less concern about social acceptance or performance. An example of this third type is a man who was preoccupied with the shape of his penis. He complained that the flesh on one side of the frenulum of the penis was flatter than on the other side. He had no concerns about his sexual performance or what his girlfriend would think if she could see that his penis was not symmetrical.

\section{Assessing suitability for therapy}

The decision to offer CBT to a person with BDD is made on the basis of a standard assessment of suitability for therapy (Box 1). Patients with a more severe disability or patients with depression may do better on a more intensive programme of CBT, for example, on a day-patient or in-patient basis, or in group therapy, which can help patients to test out their beliefs.

The very nature of BDD means that a therapist will disagree with a patient's description of the problem in terms of the exact beliefs about the patient's appearance. However, both patient and therapist can usually agree on a description of the problem as a preoccupation with appearance leading to various self-defeating behaviours. It may

Box 1 Assessing the suitability of a person for therapy

Both patient and therapist try to agree on: A description of the problem and of the goals of therapy

A formulation of the problem, i.e. an understanding of how it developed and how it is being maintained

Patient and therapist should discuss:

What the patient hopes the therapy will involve

What the patient expects therapy to involve Whether the patient's goals are realistic The estimated number of sessions required or when the therapy is to be reviewed

The frequency of sessions

The expectations of homework 
be possible to agree initially on goals such as stopping specific behaviours (e.g. skin-picking) or entering public situations that were previously avoided. Here the implicit aim is to help the patient function and do more despite his or her appearance and aesthetic standards. However, patients often have covert goals of wanting to remain excessively camouflaged in public or of changing their appearance. I specifically ask patients not to plan cosmetic surgery or dermatological treatment during therapy and to reconsider their desire for surgery after they have recovered from BDD (or at least finished therapy). In patients who are unable to enter therapy, it is to best to put the goals to one side and to concentrate on engaging the patient in a cognitive model and later negotiate the goals. Not all patients want 'therapy': some may have been forced to see a psychiatrist or a therapist by a relative or cosmetic surgeon; some are too suicidal or lacking in motivation; some will accept medication, which may act as a holding operation while trying to engage the patient in a psychological treatment.

\section{Engagement}

One method of engagement is similar to that described for hypochondriasis (Clark et al, 1998). The patient is presented with two alternative hypotheses. The first (that the patient has been following) is that he or she is 'defective' and ugly and has therefore tried hard to establish exactly what he or she looks like and to camouflage or change this appearance. The second is that the patient excessively worries about his or her appearance and makes appearance the most important aspect of identity. Patients assume a model of "what you see is what you get" in front of a mirror. An alternative model is presented: "what you see is what you have constructed" by selective attention to an internal representation of body image. The latter will depend more on the patient's mood, the meaning that they attach to appearance and the expectations that they bring to a mirror.

The therapist next moves on to a description of a cognitive-behavioural model for BDD, showing how a person with BDD becomes excessively aware of his or her appearance and giving other examples of selective attention in everyday life. Motivational interviewing can be used to focus on the consequences of the patient's preoccupation.

The therapist then asks the patient to suspend judgement and to test out the second hypothesis for the period of therapy. Engagement is usually helped by the credibility of the clinician, who validates the patient's beliefs (e.g. "what you feel about your appearance is very understandable"), rather than discounting or trivialising them (Linehan, 1993). Having reassured the patient, the clinician must then search for and reflect on the evidence for the patient's beliefs and assumptions. The clinician might recommend that patients read about BDD (e.g. Phillips, 1996, which is written for sufferers), or meet other sufferers in a patient support group or workshop at a national conference (Obsessive Action, Aberdeen Centre, 22 Highbury Grove, London N5 2EA. Tel: 0207226 4000). Patients are often extremely relieved and surprised to talk to other BDD patients.

Sometimes patients with BDD cannot be engaged in either CBT or pharmacotherapy on first presentation, and they undergo unnecessary surgery, beauty therapies, dermatological treatment or suicide attempts before finally accepting help from a mental health professional. Patients should be advised that there are always cosmetic surgeons, dermatologists and beauty therapists willing to treat them and that such treatments for BDD often result in dissatisfaction. Even if patients are satisfied with cosmetic treatments, often the preoccupation moves to a different area of the body, so that the handicap of the disorder remains (Veale, 2000). This is in marked contrast to patients without BDD who have undergone cosmetic surgery and who have good psychological benefits from the procedures.

\section{A cognitive-behavioural model of BDD}

The model of BDD shown in Fig. 1 focuses on the experience of patients when they are alone (rather than in social situations, when their behaviour is likely to follow a model similar to that of social phobia; Clark \& Wells, 1995). The model begins with the trigger of an external representation of the individual's body image, typically in front of a mirror. Alternative triggers include looking at a photograph taken when the patient was younger. The process of selective attention begins by focusing on specific aspects of the external representation (e.g. the reflection in the mirror), which leads to a heightened awareness and relative exaggeration of certain features. As a result of this process, the person with BDD constructs a distorted mental representation of his or her body image. Mirror-gazing activates idealised values about the importance of appearance and, in some patients, values about perfectionism or symmetry and thinking of the self as an aesthetic object. This leads to a negative aesthetic appraisal 
and comparisons of three different images - the external representation (usually in a mirror), the ideal body image and the distorted body image. Not surprisingly, these repeated comparisons leave the patient uncertain about his or her appearance, which encourages further mirror-gazing. The patient's desire to see exactly how he or she looks is only rewarded by looking in the mirror. However, the longer the person looks, the worse he or she feels and the more the belief of ugliness and defect is reinforced. When not looking in a mirror, the individual may focus attention on his or her internal body image and ruminate on its ugliness. There is often a marked discrepancy between the actual and the ideal body image, and this inevitably leads to depressed mood and negative thoughts.

\section{Mood and dysmorphic beliefs}

Mood changes in BDD are complex. Patients may experience anticipatory anxiety prior to mirrorgazing, when they hope they may see something different or think they will feel worse if they resist gazing. When they look in a mirror, they may experience depressed mood as they lose their hope that they might have changed. They might feel disgust as they evaluate their body image. During a long session in front of a mirror, some patients experience a dissociative state (similar to the experience of self-mutilation or bingeing). After mirror-gazing, some become angry or feel guilty for wasting so much time in front of a mirror. This judgement about being ugly appears to be closely linked to mood and is incorporated in the feedback loop of the model. Depressed mood also triggers mirror-gazing, thus creating a vicious circle. Social avoidance and social isolation also contribute to a depressed mood.

\section{The drive to change appearance}

Negative aesthetic judgement, internal aversion to the self and social anxiety drive the patient's need to change appearance. Make-up, beauty or dermatological treatments and cosmetic surgery may make physical changes, but they do not usually alter internal body image. This may lead to further disappointment and depression at the failure to meet an ideal or result in anger directed against the self or the beautician or surgeon for failing or making the appearance worse. Camouflage or cosmetic surgery inevitably lead to further mirror-gazing to evaluate its continuing efficacy and this feeds the distorted body image.

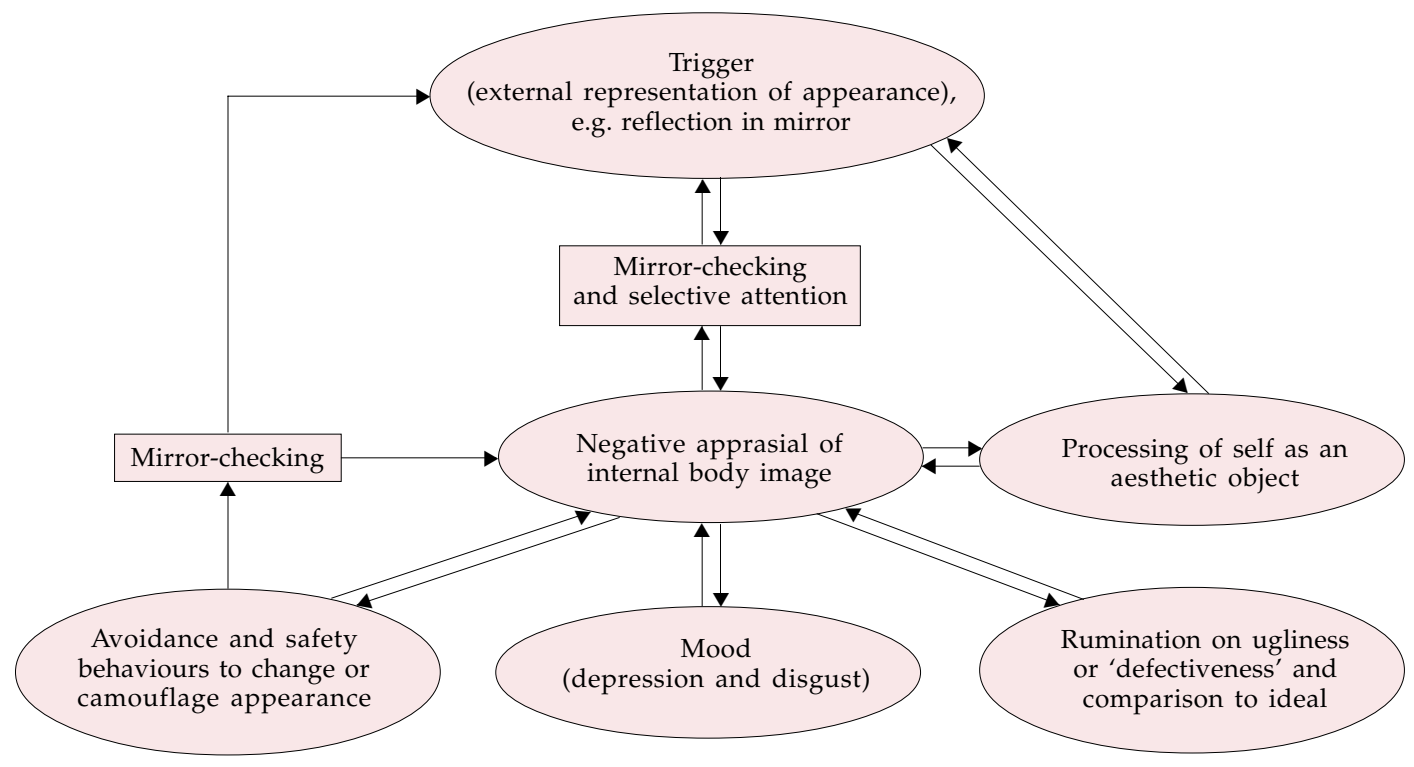

Fig. 1 A cognitive-behavioural model of body dismorphic disorder. 
Box 2 Examples of therapeutic strategies for treating body dismorphic disorder

Cognitive restructuring and behavioural experiments to test out assumptions

Reverse role-play of assumptions and values

Exposure to social situations without safety behaviour

Response prevention for compulsive behaviours such as mirror-gazing

Self-monitoring, with a tally counter, for impulsive behaviours such as skin-picking

Habit reversal for impulsive behaviours

\section{Treatment sessions}

Each patient should have an individual formulation based on the model shown in Fig. 1, which emphasises the pattern of thinking and behaviours that maintain the disorder.

Once a patient is engaged in therapy and willing to test out alternatives the therapist can chose from a variety of strategies (Box 2). Where necessary, others who are normally involved in the provision of reassurance or verification of the defect are included in a response prevention programme. They may be given instructions not to discuss requests for reassurance.

It is not possible to cover in detail here all aspects of therapy. In principle, cognitive therapy is probably more effective if it targets not the patient's beliefs about his or her appearance, but rather his or her assumptions or meaning about being 'defective' and the importance of appearance to his or her identity. This might include collecting evidence for and against assumptions such as, "If my appearance is defective then I will be unloved and alone all my life."

Values are probably best challenged by questioning their functional cost and by reducing their importance to the self in small degrees on a continuum (using a process similar to motivational interviewing in anorexia nervosa; Treasure \& Ward, 1997). A fundamental thinking error is overgeneralisation, in which the 'self' is identified only through the external appearance (the person is an aesthetic object) and all other values and selves are diminished. In this regard, a patient may be helped by the concept of 'Big I' and 'Little i': the self, or Big I, is defined by thousands of Little i's in the form of beliefs, values and characteristics since birth
(Lazarus, 1977; Dryden, 1998). The patient is therefore encouraged to focus on all the characteristics of his or her self to develop a more helpful or flexible view. Reverse role-play may be used to strengthen an alternative belief: the patient practices arguing the case for an alternative new belief while the therapist argues the case for the old beliefs (Newell \& Shrubb, 1994).

\section{Mirror-gazing}

Mirror-gazing is an early target for intervention as it feeds the selective attention on appearance. Some patients cover up or remove mirrors (previous therapists may have encouraged this). However, in my experience this can lead to the problem of mirror avoidance, and the patient is likely to maintain a distorted body image and symptoms of BDD. Furthermore, an accidental glimpse of a reflection in a mirror may be overwhelming. I think it is better that patients learn to use mirrors in a healthy way, with time limits set for various activities (e.g. putting on make-up). Patients (whether they are gazing into or avoiding them) may need some guidance on their use of mirrors (Box 3). In general, patients are encouraged to be aware of their appearance in the external reflection of a mirror, but to suspend judgement (similar to 'mindfulness'; Linehan, 1993).

Box 3 Dos and don'ts of mirror use

Use mirrors at a slight distance and use ones that are large enough to show most of the body

Focus attention on the reflection in the mirror rather than on how you feel

Use a mirror only for an agreed function (e.g. shaving, putting on make-up) and for a limited period of time

Use a variety of different mirrors and lights rather sticking to one that you 'trust'

Focus attention on the whole of your face rather than on selected areas

Do not use mirrors that magnify

Do not use ambiguous reflections (e.g. windows, the backs of compact disks or cutlery)

Do not to use a mirror when you feel depressed: try to delay essential use until you feel happier or find other things to do until the urge to mirror-gaze has passed 
When patients reduce the amount of excessive camouflage on their face others may comment on their different appearance. This requires some preparation, as the comments about being different are likely to be distorted into being ugly.

If strategies to control mirror-gazing fail, the therapist might introduce the idea of a 'response cost', in which the patient agrees to pay a hated organisation a sum of money for each prolonged check in the mirror.

\section{Pharmacotherapy}

The results of one RCT comparing clomipramine and desipramine (Hollander et al, 1999) and two case series studying fluvoxamine (Perugi et al, 1996; Phillips et al, 1998) favour SSRIs for treating BDD. Patients with delusions respond as favourably as those without (Phillips et al, 1998). Contrary to popular belief, there is no evidence for the efficacy of pimozide or antipsychotics as the sole treatment, even in patients with delusions. The case reports described by Riding \& Munro (1975) included cases of delusions of infestation and body odour and dysmorphic delusions. Although there have been no RCTs of an SSRI against an antipsychotic, Phillips (1998) reported that in 113 trials an SSRI was of benefit in $54 \%$ of cases, compared to $2 \%$ in 83 antipsychotic trials. SSRIs are especially indicated when BDD patients have a significantly depressed mood, are at risk of suicide, or there is a long waiting list for CBT. Treatment guidelines are the same as for obsessive-compulsive disorder (OCD) - an SSRI in the highest dose for a prolonged period of time.

Opinions vary on treatment-resistant cases. They may require an alternative SSRI or clomipramine. Patients that remain resistant might be helped by either: (a) an SSRI in very high doses; (b) an SSRI in combination with a very low dose of an antipsychotic as an adjunct; or (c) serotonin augmentation strategies such as buspirone. As with OCD, there is probably a high risk of relapse on discontinuation of an SSRI. As yet there are no RCTs comparing CBT with an SSRI, but there is no suggestion that a combined approach is unhelpful. Indeed, maintaining a stable mood and reducing self-consciousness may have a synergistic benefit.

\section{References}

American Psychiatric Association (1994) Diagnostic and Statistical Manual of Mental Disorders (4th edition) (DSMIV). Washington, DC: APA.
Clark, D. M. \& Wells, A. (1995) A cognitive model of social phobia. In Social Phobia - Diagnosis, Assessment, and Treatment (eds R. G. Heimberg, M. R. Liebowitz, D. Hope, et al), pp. 69-93. Guilford Press: New York.

_-, Salkovskis, P. M., Hackmann, A., et al (1998) Two psychological treatments for hypochondriasis. A randomised controlled trial. British Journal of Psychiatry, $173,218-225$.

Dryden, W. (1998) Developing Self-Acceptance. Chichester: John Wiley \& Sons.

Faravelli, C., Salvatori, S., Galassi, F., et al (1997) Epidemiology of somatoform disorders: a community survey in Florence. Social Psychiatry and Psychiatric Epidemiology, 32, 24-29.

Gomez Perez, J. C., Marks, I. M. \& Gutierrez Fisac, J. L. (1994) Dysmorphophobia: clinical features and outcome with behavior therapy. European Psychiatry, 9, 229-235.

Hollander, E., Allen, A., Kwon, J., et al (1999) Clomipramine vs desipramine crossover trial in body dysmorphic disorder: selective efficacy of a serotonin reuptake inhibitor in imagined ugliness. Archives of General Psychiatry, 56, $1033-1042$.

Lazarus, A. (1977) Towards an ego-less state of being. In Handbook of Rational Emotive Therapy (Vol. 1) (eds A. Ellis \& R. Grieger), pp. 113-118. New York: Springer.

Linehan, M. M. (1993) Skills Training Manual. Guilford Press.

Newell, R. \& Shrubb, S. (1994) Attitude change and behaviour therapy in body dysmorphic disorder: two case reports. Behavioural and Cognitive Psychotherapy, 22, 163-169.

Neziroglu, F. \& Yaryura Tobias, J. A. (1993) Exposure, response prevention, and cognitive therapy in the treatment of body dysmorphic disorder. Behavior Therapy, 24, 431438

Perugi, G., Giannotti, D., Di Vaio, S., et al (1996) Fluvoxamine in the treatment of body dysmorphic disorder (dysmorphophobia). International Clinical Psycho-pharmacology, 11, 247-254.

Phillips, K. (1996) The Broken Mirror - Understanding and Treating Body Dysmorphic Disorder. New York: Oxford University Press.

Phillips, K. A. (1998) Body dysmorphic disorder: clinical aspects and treatment strategies. Bulletin of the Menninger Clinic, 62, A33-A48.

- (2000) Quality of life for patients with body dysmorphic disorder. Journal of Nervous and Mental Disease, 188, 170175

-, Dwight, M. M. \& McElroy, S. L. (1998) Efficacy and safety of fluvoxamine in body dysmorphic disorder. Journal of Clinical Psychiatry, 59, 165-171.

Riding, J. \& Munro, A. (1975) Pimozide in the treatment of monosymptomatic hypochondriacal psychosis. Acta Psychiatrica Scandinavica, 53, 23-30.

Rosen, J. C., Reiter, J. \& Orosan, P. (1995) Cognitive-behavioral body image therapy for body dysmorphic disorder. Journal of Consulting \& Clinical Psychology, 63, 263-269.

Sarwer, D. B., Wadden, T. A., Pertschuk, M. J., et al (1998) Body image dissatisfaction and body dysmorphic disorder in 100 cosmetic surgery patients. Plastic and Reconstructive Surgery, 101, 1644-1649.

Treasure, J. L. \& Ward, A. (1997) A practical guide to the use of motivational interviewing. European Eating Disorders Review, 5, 102-114.

Veale, D. (2000) Outcome of cosmetic surgery and 'DIY' surgery in patients with body dysmorphic disorder. Psychiatric Bulletin, 24, 218-220.

— \& Riley, S. (2001) Mirror, mirror on the wall, who is the ugliest of them all? The psychopathogy of mirror gazing in body dysmorphic disorder. Behaviour Research and Therapy (in press).

- Gournay, K., Dryden, W., et al (1996a) Body dysmorphic disorder: a cognitive behavioural model and pilot randomised controlled trial. Behaviour Research and Therapy, 34, 717-729.

-, Boocock, A., Gournay, K., et al (1996b) Body dysmorphic disorder. A survey of fifty cases. British Journal of Psychiatry, 169, 196-201. 
Wilhelm, S., Otto, M. W., Lohr, B., et al (1999) Cognitive behavior group therapy for body dysmorphic disorder: a case series. Behaviour Research and Therapy, 37, 71-75. World Health Organziation (1992) The ICD-10 Classification of Mental and Behavioural Disorders. Geneva: WHO.

\section{Multiple choice questions}

1. Typical behaviours in BDD include:
a combing and parting the hair until it is exactly symmetrical
b skin-picking
c wearing excessive make-up 24 hours a day
d hair-plucking
e staring at reflection in compact disks or cutlery.

2. BDD patients are drawn to mirrors because they:
a are vain
b hope that they will look different
c want to know exactly how they look
d think they will feel worse if they resist gazing
e are narcissistic.

3. Values that are usually extremely important to BDD patients include:
a perfectionism
b symmetry
c social acceptance
d achievement
e health.

4. Cognitive-behavioural strategies in BDD may include:

a reverse role-play for beliefs

b prolonged self-exposure to mirrors

c self-monitoring and habit reversal for skinpicking

d reassurance by relatives that patient's appearance is all right

e validation of patient's beliefs.

5. First-line pharmacological treatment of BDD includes:

a an antipsychotic (e.g. pimozide)

b an SSRI (e.g. fluoxetine)

c a noradrenaline reuptake inhibitor (e.g. reboxetine)

d a monoamine oxidase inhibitor

e clomipramine.
MCQ answers

$\begin{array}{llllllllll}\text { 1 } & & \text { 2 } & & \text { 3 } & & 4 & & \text { 5 } & \\ \text { a } & \text { T } & \text { a } & \text { F } & \text { a } & \text { T } & \text { a } & \text { T } & \text { a } & \text { F } \\ \text { b } & \text { T } & \text { b } & \text { T } & \text { b } & \text { T } & \text { b } & \text { F } & \text { b } & \text { T } \\ \text { c } & \text { T } & \text { c } & \text { T } & \text { c } & \text { T } & \text { c } & \text { T } & \text { c } & \text { F } \\ \text { d } & \text { F } & \text { d } & \text { T } & \text { d } & \text { F } & \text { d } & \text { F } & \text { d } & \text { F } \\ \text { e } & \text { T } & \text { e } & \text { F } & \text { e } & \text { F } & \text { e } & \text { T } & \text { e } & \text { T }\end{array}$

